\title{
EFFECTS OF S-PROCESS BRANCHINGS ON STELLAR AND METEORITIC ABUNDANCES*
}

E. B. Norman, K. T. Lesko, S. G. Crane, R. M. Larimer Nuclear Science Division, Lawrence Berkeley Laboratory Berkeley, CA 94720 U.S.A., and

A. E. Champagne

Physics Department, Princeton University

Princeton, NJ 08544 U.S.A.

ABSTRACT. The level scheme and electromagnetic properties of $148 \mathrm{pm}$ have been studied using ${ }^{149} \mathrm{Sm}(\mathrm{d}, 3 \mathrm{He})$ and $148_{\mathrm{Nd}}\left(\mathrm{p}, \mathrm{n}_{\gamma}\right)$ reactions. Combining these measurements with estimates for E2/M1 decay branching ratios leads to the tentative conclusion that $148 \mathrm{Pmg}, \mathrm{m}$ are in thermal equilibrium during the s-process. The branch at $148 \mathrm{Pm}$ then leads to an inferred s-process neutron density of $3 \times 10^{8} \mathrm{~cm}^{-3}$.

At a number of points along the path of the slow neutron-capture process (s-process), nuclei are encountered which are sufficiently long-lived that neutron capture on these isotopes can compece with their normal beta decays. That such effects actually occur has been inferred from observations of both stellar and meteoritic elemental and isotopic abundances. The details of these s-process branchings depend sensitively on stellar environmental factors such as temperature, matter density, and neutron density. Thus, these branch points can provide specific information on the stellar conditions under which the s-process occurs.

Tomk in and Lambert 1 examined the effects of the s-process branch at $85 \mathrm{Kr}$ on the elemental abundances they observed in the barium star HR 774. From their studies, it was concluded that in this star the s-process neutron density was in the range $10^{6} \leqslant N(n) \leqslant 10^{7} \mathrm{~cm}^{-3}$.

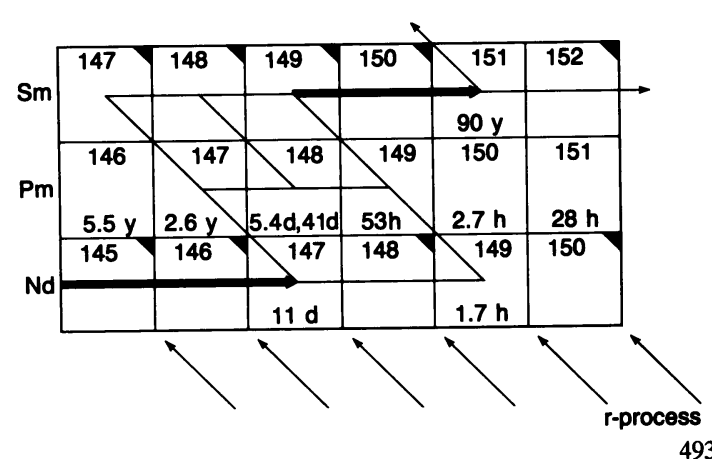

Figure 1. The s-process path through the mass region $145 \leqslant A \leqslant 152$. Stable isotopes are indicated by shaded triangles. Beta decay half-lives of unstable isotopes are indicated. 
Kappeler ${ }^{2}$ has investigated the s-process branchings at $151 \mathrm{Sm}$, $185 \mathrm{~W}$, and $204 \mathrm{Tl}$. From measurements and calculations of neutroncapture cross sections and measurements of meteoritic abundances, he has inferred an s-process neutron density on the order of $3 \times 10^{8} \mathrm{~cm}^{-3}$. Furthermore, a possible trend toward the requirement of higher neutron densities for the producton of the heavier nuclei can be seen in Käppeler's results

The branch at $148 \mathrm{pm}$ illustrated in Fig. 1 offers another opportunity to determine the s-process neutron density. 3 The inferred value of this quantity depends sensitively on whether or not $148 \mathrm{Pm}, \mathrm{m}$ are in thermal equilibrium at the temperatures found in the helium-burning zones of red-giant stars. A possible mechanism by which such equilibration could occur is shown in Fig. 2. To decide this issue, the positions and decay modes of $148 \mathrm{Pm}$ excited states must be known. Using the Princeton Q3D spectrograph we have observed the large number of low-lying $148 \mathrm{Pm}$ excited states shown in Fig. 3. At LBL'S 88-Inch Cyclotron we have used $\gamma$-ray singles and $\gamma-\gamma$ coincidences from the $148 \mathrm{Nd}(p, n) 148 \mathrm{Pm}$ reaction to study the decay modes of these levels. A large number of $\gamma$-ray lines between 75 and $400 \mathrm{keV}$ have been observed and coincidence relations for many transitions have been establijshed. As yet, no direct link between $148 \mathrm{pmg}, \mathrm{m}$ (such as that shown in Fig. 2) has been observed. However, combining the observed high level density and $\gamma$-ray transitions with theoretical estimates of E2/M1 decay branching ratios leads to the tentative conclusion that $148 \mathrm{Pm}, \mathrm{m}$ are in thermal equilibrium during the s-process. The inferred neutron density using this branch point is then $3 \times 10^{8} \mathrm{~cm}^{-3}$.

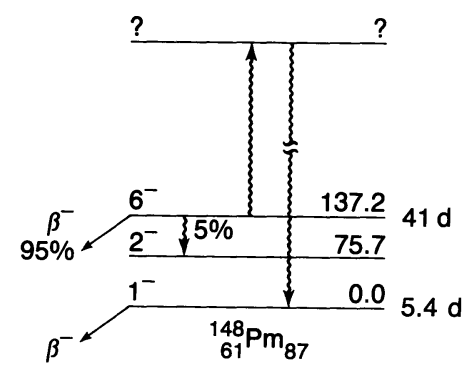

Figure 2. Partial level scheme of $148 \mathrm{Pm}$ illustrating the possibility of photoexcitation of $148 \mathrm{Pm}^{\mathrm{m}}$ to a higher lying state that decays to $148 \mathrm{Pmg}$.

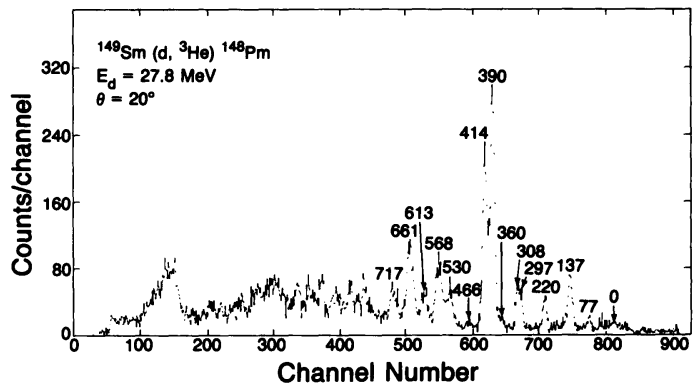

Figure 3. Spectrum observed from the ${ }^{149} \mathrm{Sm}\left(\mathrm{d},{ }^{3} \mathrm{He}\right){ }^{148 \mathrm{Pm}}$ reaction at 20 degrees. Energies of $148 \mathrm{Pm}$ excited states are given in keV.

\section{REFERENCES}

*Supported by U.S. Dept. of Energy contract No. DE-AC03-76SF00098, and U.S. National Science Foundation contract No. PHY83-04977.

1. J. Tomk in and D. L. Lambert. Ap. J. 273, 722 (1983).

2. F. Käppeler, Proc. 2nd Wrkshp. NucT. Astrophys., MPA 90, (1983) p.82.

3. F. Käppeler, AIP Conf. Proc. 125, 715 (1985). 